BULLETIN OF THE

AMERICAN MATHEMATICAL SOCIETY

Volume 79, Number 1, January 1973

\title{
SUBMERSIONS FROM SPHERES
}

BY R. ESCOBALES, JR.

Communicated by S. S. Chern, August 9, 1972

1. Let $M$ and $B$ be Riemannian manifolds with $M$ connected and complete. Assume $\pi$ and $\bar{\pi}$ are Riemannian submersions from $M$ onto $B$ so that the fibers of these two submersions are totally geodesic. $\pi$ and $\bar{\pi}$ are said to be equivalent provided there exists an isometry $f$ of $M$ which induces an isometry $\underline{f}$ of $B$ so that the following diagram is commutative.

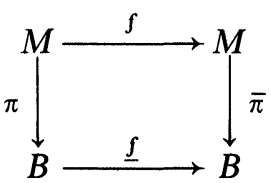

We call the pair $(f, f)$ a bundle isometry of $\pi$ and $\bar{\pi}$. Now set $\pi=\bar{\pi}$. $\pi$ is homogeneous if for every $p, q \in M$ there exists a bundle isometry $(f, f)$ of $\pi$ with $f(p)=q$.

In what follows $S^{m}$ denotes the unit $m$ sphere while $S^{q}(r)$ denotes a $q$ sphere of radius $r . K_{*}\left(P_{X} Y\right)$ denotes the curvature of a 2 plane in $B$ spanned by $X$ and $Y$.

For a Riemannian submersion $\pi: M \rightarrow B$, O'Neill [10] has defined a tensor $A$ which we call the integrability tensor of $\pi$. If $A \equiv 0$, then the horizontal distribution (the distribution complementary to the fibers in the tangent space of $M$ ) is integrable. In general we will follow the notation of [10]. We now state our first result. Complete proofs are found in [4].

THEOREM 1.1. Let $\pi: S^{m} \rightarrow B$ be a Riemannian submersion with totally geodesic fibers. Assume $1 \leqq \operatorname{dim}$ fiber $\leqq m-1$. Then as a fiber bundle $\pi$ is one of the following types:
(a) $\begin{aligned} S^{1} \rightarrow & S^{2 n+1} \\ & \downarrow \pi\end{aligned}$
(b) $\begin{aligned} & S^{3} \rightarrow S^{4 n+3} \\ & \downarrow \pi\end{aligned}$
$C p(n)$ for $n \geqq 2$
$Q p(n)$ for $n \geqq 2$
(c) $\begin{aligned} S^{1} \rightarrow & S^{3} \\ & \downarrow \downarrow \pi\end{aligned}$
(d) $\begin{aligned} S^{3} \rightarrow & S^{7} \\ & \downarrow \pi\end{aligned}$
(e) $\begin{aligned} S^{7} \rightarrow & S^{15} \\ & \downarrow \pi\end{aligned}$
$S^{2}\left(\frac{1}{2}\right)$
$S^{4}\left(\frac{1}{2}\right)$
$S^{8}\left(\frac{1}{2}\right)$

In cases $(a)$ and $(b) B$ is isometric to complex projective $n$-space and quaterionic $n$-space respectively with $1 \leqq K_{*}\left(P_{X} Y\right) \leqq 4$. In cases $(c),(d)$ and $(e)$ $B$ is isometric to a sphere of curvature 4 .

AMS (MOS) subject classifications (1970). Primary 53C20; Secondary 55F05.

Key words and phrases. Riemannian submersion, equivalent bundle isometry, integrability tensor. 
Any two submersions both of type $(a),(b)$, or (c) are equivalent and any submersion in one of these three cases is homogeneous.

Proposition 1.2. Let $\pi: M \rightarrow B$ be a Riemannian submersion with totally geodesic fibers. If $M$ is $\delta$ pinched, $B$ is $\delta / 4$ pinched.

ProOF OF 1.2. We let $X$ and $Y$ be any orthonormal horizontal vectors. If $A_{X} Y \neq 0$ we show $\left\|A_{X} Y\right\| \leqq 1$ using the relations of O'Neill and exploiting the skew-symmetry of $A_{X}$. Dropping the assumption $A_{X} Y \neq 0$ and appealing to Corollary 1 of O'Neill [10] we show $\delta \leqq K_{*}\left(P_{X} Y\right)$ $=1+3\left\|A_{X} Y\right\|^{2} \leqq 4$. If $M=S^{m}$ then our result implies $B$ is $1 / 4$ pinched.

Proof of 1.1. $\pi$ is a fiber bundle and $B$ is complete by the result of Hermann [6]. Now the only complete totally geodesic subspaces of spheres are spheres. By a result of Adem [2] it follows $m$ and the fiber dimension are odd. A fiber homotopy sequence shows $B$ is simply connected by our proposition $B$ is $1 / 4$ pinched. Hence Berger's theorem $([3],[7])$ implies $B$ is isometric to one of the projective spaces or is homeomorphic to a sphere. Adem's result combined with standard fiber homotopy sequence arguments yield that if $B$ is $C p(n)$ or $Q p(n)$ with $n \geqq 2$ then the only submersions are of types (a) or (b). If $B$ is $C_{a}^{2}(P)$, the Cayley two plane, then by Adem's result $m=31$ and dim fiber $=15$. Thus $\pi_{i}\left(S^{31}\right)$ $\rightarrow \pi_{i}\left(C_{a}^{2}(P)\right) \rightarrow \pi_{i-1}\left(S^{15}\right) \rightarrow \pi_{i-1}\left(S^{31}\right)$ yields $\pi_{i}\left(C_{a}^{2}(P)\right)=0$ for $1 \leqq i \leqq 15$. The Hurewicz theorem implies $C_{a}^{2}(P)$ is a homology 16 sphere which it is not. Hence the only submersions over projective spaces $\left(\neq S^{2}, S^{4}, S^{8}\right)$ are of types (a) and (b).

From Adam's result [1] it follows that the only fiber bundles with total space, fiber and base each homeomorphic to spheres are cases (c), (d) and (e) of the theorem. Now in our situation both total space and fiber are unit spheres. To show $B$ is isometric to a sphere in each case we again take horizontal orthonormal vectors $X$ and $Y$. Our procedure, which handles the three cases simultaneously, allows us to assert $\left\|A_{X} Y\right\| \geqq 1$. But $\left\|A_{X} Y\right\| \leqq 1$ as in the proof of 1.2. From O'Neill's equation it follows $K_{*}\left(P_{X} Y\right)=1+3\left\|A_{X} Y\right\|^{2}=4$.

2. To complete the proof of Theorem 1.1 we need another result which is of independent interest.

THEOREM 2.1. For $i=1,2$ let $\pi_{i}$ be Riemannian submersions with totally geodesic connected fibers from $M$ onto $B$. Assume $M$ is connected and complete. Suppose $f$ is an isometry of $M$ which satisfies the following two properties for one point $p \in M$.

(1) $f_{* p}$-the differential of $f$ at $p$-maps $H_{1 p}$ onto $H_{2 f(p)}$ where $H_{i}$ denotes the horizontal distribution of $\pi_{i}$. 
(2) Denote the tangent space of $M$ at $p$ by $T_{p} M$. If $E, F \in T_{p} M$, then $f_{*} A_{1 E} F=A_{2 f_{*} E} f_{*} F$ where $A_{i}$ is the integrability tensor of $\pi_{i}$.

Then $f$ is fiber preserving and induces $f$ of $B$ so that $(f, f)$ is a bundle isometry of $\pi_{1}$ and $\pi_{2}$.

COROLlary 2.2. Any two submersions $\pi$ and $\bar{\pi}$ which satisfy the hypotheses of 1.1 and are of the same type are equivalent provided they are of type (a), (b), or (c) in 1.1. Any submersion $\pi$ in one of these three classes is homogeneous.

Corollary 2.2 completes the proof of 1.1 .

SKETCH OF 2.1. Take a geodesic $\gamma$ in $B$ with $\gamma(0)=\pi_{1}(p)$. Denote the fiber over $\gamma(t)$ by $S_{\gamma(t)}$. Then $f\left(S_{\gamma(t)}\right)$ coincides with $\pi_{2}$ fiber through $f(p)$ by (1) in the theorem and a result on connected totally geodesic subspaces. It follows for every $q \in S_{\gamma(0)}, f_{*} H_{1 q}=H_{2 f(q)}$ since these subspaces are complementary to the tangent spaces of the fibers. The family $\{\tilde{\gamma}\}$ consisting of the $\pi_{1}$ horizontal lifts of $\gamma$ defines an isometry between $S_{\gamma(0)}$ and $S_{\gamma(t)}([6],[8])$. Call this isometry $F_{\gamma(t)}$.

The family $\{f \circ \tilde{\gamma}\}$ are horizontal geodesics since $f \circ \tilde{\gamma}(0) \in H_{2 f(q)}$ for $q \in S_{\gamma(0)}$ and hence is everywhere horizontal [11]. It can be shown $\{f \circ \tilde{\gamma}\}$ are the $\pi_{2}$ horizontal lifts of a geodesic $v$ of $B$ with $v(0)=\pi_{2} f(p)$. This family defines an isometry between the $\pi_{2}$ fibers over $v(0)$ and $v(t)$ denoted by $S_{v(0)}$ and $S_{v(t)}$ respectively. Denote this isometry by $F_{v(t)}$. In fact $f \circ F_{\gamma(t)}=$ $F_{v(t)}$. It follows $f\left(S_{\gamma(t)}\right)=S_{v(t)}$. Since $B$ is geodesically complete, $f$ is fiber preserving. Routine arguments show the map $f$ of $B$ induced by $f$ is an isometry.

We omit the proof of 2.2 which is lengthy. Substantial use of the curvature equations in $[\mathbf{1 0}]$ is required. I do not know if results analogous to those of 2.2 obtain for submersions of types (d) and (e) in 1.1.

REMARK. A result similar to the first part of 1.1 was obtained by Nagano [9] for homogeneous sphere bundles. Since we make no assumption about homogeneity our proof relies rather on the properties of submersion metrics. In 1.1 of course we assume connected fibers.

\section{BIBLIOGRAPHY}

1. J. F. Adams, On the non-existence of elements of Hopf invariant one, Ann. of Math. (2) 72 (1960), 20-104. MR 25 \#4530.

2. J. Adem, Relations on iterated reduced powers, Proc. Nat. Acad. Sci. U.S.A. 39 (1953), 636-638. MR 15, 53.

3. M. Berger, Sur quelques variétés riemanniennes suffisamment pincées, Bull. Soc. Math. France 88 (1960), 57-71. MR 24 \# A3606.

4. R. Escobales, The differential geometry of Riemannian submersions, Notre Dame Dissertation, 1972.

5. A. Gray, Pseudo-Riemannian almost product manifolds and submersions, J. Math. Mech. 16 (1967), 715-737. MR 34 \# 5018.

6. R. Hermann, A sufficient condition that a map of Riemannian manifolds be a fiber bundle, Proc. Amer. Math. Soc. 11 (1960), 236-242. MR 22 \# 3006. 
7. S. Kobayashi and K. Nomizu, Foundations of differential geometry. Vols. I, II, Interscience, New York, 1963, 1969. MR 27 \# 2945.

8. T. Nagano, On fibred Riemann manifolds, Sci. Papers Coll. Gen. Ed. Univ. Tokyo 10 (1960), 17-27. MR 28 \# 560.

9. Math. J. 15 (1959), 29-55. MR 21 \# 7522.

10. B. O'Neill, The fundamental equations of a submersion, Michigan Math. J. 13 (1966), 459-469. MR 34 \# 751.

11. - Submersions and geodesics, Duke Math. J. 34 (1967), 363-373. MR 35 \# 7265 .

Department of Mathematics, University of Notre Dame, Notre Dame, Indiana 46556 